\title{
Kernos
}

Revue internationale et pluridisciplinaire de religion grecque antique

13 | 2000

Varia

\section{Giovanni CASADIO, Il vino dell'anima. Storia del culto di Dioniso a Corinto, Sicione, Trezene}

\section{Isabelle Tassignon}

\section{OpenEdition \\ Journals}

\section{Édition électronique}

URL : http://journals.openedition.org/kernos/1315

DOI : 10.4000/kernos. 1315

ISSN : 2034-7871

\section{Éditeur}

Centre international d'étude de la religion grecque antique

Édition imprimée

Date de publication : 1 janvier 2000

ISSN : 0776-3824

\section{Référence électronique}

Isabelle Tassignon, "Giovanni casadıo, II vino dell'anima. Storia del culto di Dioniso a Corinto, Sicione,

Trezene », Kernos [En ligne], 13 | 2000, mis en ligne le 16 juin 2011, consulté le 24 septembre 2020

URL : http://journals.openedition.org/kernos/1315; DOI : https://doi.org/10.4000/kernos.1315 
l'image traditionnelle qu'on s'est faite jusqu'ici d'Apollon. Il n'est pas sûr du tout qu'à la longue ce dieu marcheur et égorgeur soit de nature à faire bâiller moins de classes que ne le fait aujourd'hui, paraît-il, le dieu de la sagesse dès qu'est prononcé son nom. Mais ne préjugeons pas ici de l'avenir ! Il n'est pas sûr non plus que la méthode annoncée ait tenu toutes ses promesses. C'est le cas notamment du dessein de définir Apollon dans la texture d'un système polythéiste. Il y a bien çà et là, d'utiles remarques à propos des rapports entre Apollon et certains de ses congénères, comme Poseidon et Hestia, mais il n'apparaît pas que toutes les possibilités qu'offraient les textes utilisés, à commencer par l'Hymne boméri$q u e$, aient été de ce point de vue exploitées. Tout compte fait, on n'est pas, de ce point de vue, si éloigné du genre monographique que critique volontiers l'A. Et que dire ici de son idée d'expérimentation? Il en convient : nous n'avons pas le pouvoir de faire se répéter les phénomènes observés comme on le fait dans un laboratoire, ni non plus de modifier les conditions de l'expérience. Dès lors, le terme de comparaison qui conviendrait le mieux en l'occurrence ne serait-il pas le travail méthodique qu'on opère sur un champ de fouilles lorsqu'on s'efforce, par essais et erreurs, de reconstituer à partir des éléments mis au jour tantôt des objets, tantôt des ensembles plus importants et plus complexes comme sont les sanctuaires? Il y a bien un peu de cela dans la méthode préconisée qui consiste à observer comment réagissent les dieux par rapport à tels objets concrets, tels gestes, telles situations, pour y découvrir, sous-jacente, une sorte de logique. $\grave{A}$ noter toutefois que l'archéologue, pour sa part, a commencé par noter soigneusement les strates d'où proviennent les objets récoltés; il n'aurait pas idée de réunir d'emblée, en vue de reconstituer un vase, des fragments découverts à des niveaux fort éloignés. Se pourrait-il que, pour faire réagir des dieux à des objets, à des situations, on soit autorisé à les considérer dans un éternel présent, comme si, avec le temps, rien ne changeait autour d'eux et comme si eux-mêmes ne faisaient pas l'objet d'appréhensions différentes ? Il y aurait là, parmi d'autres, une question qu'on aimerait poser à $M$. Detienne.

Peut-être ne tardera-t-on pas à l'obtenir car cet Apollon ne sera pas le dernier ouvrage du genre, l'A. méditant à présent, ainsi qu'il l'annonce en conclusion, d'expérimenter dans l'épais dossier de la configuration, ô combien complexe, que forment ensemble les demi-frères Apollon et Dionysos. Nul n'y est sans doute mieux préparé que lui; on souhaitera seulement que l'investigation n'ait pas l'ampleur du Zeus de Cook!

André Motte

(Université de Liège)

Giovanni Casadio, Il vino dell'anima. Storia del culto di Dioniso a Corinto, Sicione, Trezene, Roma, Editrice "il Calamo", 1999. 1 vol. $17 \times 24 \mathrm{~cm}, 231 \mathrm{p}$. (Biblioteca di Storia delle Religioni, 1). ISBN : 88-86148-54-2.

Après sa Storia del culto di Dioniso in Argolide, l'A. a réuni dans cet ouvrage plusieurs contributions ayant pour thème général Dionysos. Dans le long prologue intitulé «Dioniso e il vino », l'A. tente de montrer que les liens qui unissent Dionysos au vin remontent à la plus haute antiquité et qu'il apparaîtrait déjà comme dieu du vin dans les tablettes en Lin. B. Contrairement à l'opinion défendue par U. von Wilamowitz-Møellendorff et d'autres (O. Kern, M. Müller, M.P. Nilsson, H. Jeanmaire) pour qui Dionysos serait primitivement un dieu de la végétation se spécialisant ultérieurement dans le vin, celui-ci constituerait, dès le milieu du $2^{\mathrm{e}}$ millénaire, une image synecdotique du tempérament profondément ambivalent de Dionysos. L'A. se fonde sur la tablette en linéaire B PK Xa 1419 où cohabitent autour du vin (wo-no) un mot qui en dérive (wo-no-wa-ti-si) men- 
tionné au recto et le nom de Dionysos (Di-wo-nu-so) écrit au verso. Sur ce témoignage d'interprétation délicate, je suivrais plutôt l'attitude prudente de Ruth Palmer (Wine in the Mycenaean Palace economy, Liège, 1994, p. 62 [=Aegeum, 10]) pour qui l'on ne peut rien déduire de certain de deux noms que séparent plusieurs lignes. Outre ce document du $2^{\mathrm{e}}$ millénaire, témoignages littéraires (Hésiode et Homère) et iconographiques attesteraient de liens forts entre Dionysos et le vin. Au nombre de ces représentations, il y a les reliefs dits « de la visite au poète " où Dionysos est figuré ivre, en marche, et la série des représentations qui montrent Dionysos accomplissant une libation aux pieds d'une statue. Ici, une typologie des motifs, un classement chronologique des ouvres choisies et une exégèse plus approfondie de certains documents auraient peut-être donné davantage de portée à l'argumentation; néanmoins, ces images sont difficiles à utiliser car d'époque classique ou hellénistique, elles sont beaucoup plus tardives que les documents littéraires les plus anciens témoignant d'un éventuel lien entre Dionysos et le vin.

Dans une seconde partie de l'ouvrage, l'A. aborde les cultes de Dionysos à Corinthe, Sicyone et Trézène par le biais des témoignages littéraires, en se faisant largement l'écho de Pausanias. Le récit étiologique des statues de culte de Corinthe donné par Pausanias (II, 2,6) est pour l'A. l'occasion de réaliser ce qui constitue le meilleur état de la question paru jusqu'ici sur les liens entre Dionysos et l'arbre, et d'une manière générale, de faire le point sur les manifestations de dendrolâtrie en contexte dionysiaque. Mettant à l'épreuve les hypothèses jadis formulées par U.T. Bezerra de Meneses et É. Coche de la Ferté pour qui l'arbre était l'une des formes de Dionysos, l'A. montre de manière convaincante que rien ne permet d'identifier le dieu à l'arbre, mais que l'arbre est le lieu privilégié d'épiphanie de Dionysos; on pourrait ajouter à sa démonstration, qu'à Magnésie du Méandre, c'est dans un platane arraché par le vent que le statue de Dionysos apparaît (O. Kern, Die Inschriften von Magnesia am Maeander, Berlin, 1900, $\mathrm{n}^{\circ}$ 215), et qu'il a pu à l'origine, être considéré comme une représentation du dieu, ce qui expliquerait qu'à Thèbes, on ait honoré Dionysos Cadmeios, apparu aux hommes sous la forme d'un morceau de bois tombé du ciel (Pausanias, IX, 12, 3), et qu'à Corinthe, on ait adoré l'arbre dans lequel Penthée avait subi son martyre; on en aurait fait, toujours selon Pausanias, deux statues de culte, désignées l'une sous le nom de Dionysos Baccheios, l'autre sous celui de Dionysos Lyseios. Toutes deux étaient dorées, à l'exception de leur visage, peint en rouge. Ce détail offre l'opportunité à l'A. de s'attarder à la symbolique de la couleur rouge du cinnabre, souvent utilisée pour les représentations statuaires de Dionysos : symbole du sang, cette couleur à la fois apotropaïque et funeste renverrait une fois encore à la nature complexe d'un dieu ambivalent. Cette couleur gagnerait peutêtre à être envisagée aussi dans ses rapports avec l'or qui recouvre certaines statues du dieu.

Au demeurant, les cultes de Dionysos Baccheios et Lyseios n'étaient pas l'apanage de Corinthe; ils existaient également à Sicyone. Pausanias raconte (II, 7, 5-6), de manière très allusive, qu'au cours de dromena annuels qui avaient lieu en secret, on introduisait régulièrement ces deux statues. Selon l'interprétation prônée par M.P. Nilsson, la procession décrite aurait été une simple fête privée de caractère orgiaque. L'A. réagit contre cette tendance à la banalisation; il analyse le rituel où les deux statues (Dionysos Baccbeios et Lyseios) rituellement conduites l'une après l'autre comme l'image de l'introduction successive des deux formes de culte de Dionysos. Le culte de Dionysos Lyseios serait postérieur à celui de Baccheios et aurait été, si l'on en croit l'aition, introduit tardivement depuis Thèbes par un certain Phanès, personnage qui apparaît aussi à Thèbes. À Corinthe et Sicyone, Delphes a sans doute joué un rôle décisif dans l'exportation 
d'une des formes thébaines du culte de Dionysos, celui de Dionysos Luseios. En d'autres termes, on trouverait dans ces localités, des formes de culte dionysiaques identiques à celles que l'on trouve à Thèbes. Par contre, le culte de Dionysos Baccheios, quoique très répandu, n'est, à ce jour, pas attesté comme tel à Thèbes; cette absence empêche l'A. d'y trouver l'exact équivalent des cultes de Dionysos Baccheios et Lusios. Ici, l'A. semble solliciter trop les témoignages littéraires, notamment dans le cas de la statue thébaine que Pausanias donne pour être une Sémélé, mais qui serait selon l'A. celle d'un Dionysos Baccbeios, un dieu efféminé; or à Thèbes, le culte de Sémélé aurait toute raison d'être puisque la ville passait pour être sa patrie. Aux cas étudiés par l'A., on pourrait joindre utilement celui de Magnésie du Méandre, mentionné allusivement, où le culte dionysiaque fut fondé - ou plutôt refondé - au in ${ }^{\text {e }}$ siècle avant J.-C., par l'importation, depuis Thèbes, de trois thiases sur l'injonction de l'oracle de Delphes. Cette importance de l'oracle dans la fondation d'un culte de Dionysos pose de façon aiguë le problème des rapports entre Dionysos et Apollon, propriétaire de l'oracle.

Sémélé est au centre des réflexions de l'A. dans le chapitre consacré à Trézène. Trézène, comme Delphes et Lerne, serait le lieu d'une anagôgé de Sémélé. Si l'on en croit Pausanias (II, 31, 2), dans le temple d'Artémis Sôteira, s'élevaient des autels aux dieux infernaux; c'est à cet endroit que Dionysos aurait enlevé sa mère de l'Hadès pour lui faire connaître l'apothéose. L'A. reprend ici l'étude systématique du personnage de Sémélé, personnage mythologique mineur mais qui en certains lieux semble avoir fait l'objet d'un véritable culte, comme à Thèbes, Delphes (fête de Septerion et de l'Hérö̈s) ainsi qu'en plusieurs cités d'Asie Mineure (Pergame, Cyzique, et encore Magnésie du Méandre). Réexaminant la théorie de P. Kretschmer, il montre que l'hypothèse d'une Sémélé qui aurait été une ancienne déesse-terre ne doit pas être rejetée, d'autant qu'elle se trouve associée à celle d'un dieu chthonien, qui reparaît régulièrement. Au terme d'une démonstration convaincante, il observe qu'en chaque cas, le vocabulaire s'accorde à décrire une anagôge, une remontée depuis l'Hadès d'une mortelle devenue divine par l'action d'un fils immortel; cette forme d'idéologie sera particulièrement amplifiée en Asie Mineure, et notamment dans le royaume de Pergame : plusieurs témoignages - au nombre desquels les stylopinakia du temple d'Apollonis à Cyzique - évoquent la propagande développée autour de Dionysos "sauveur ", le dieu qui donna l'immortalité à sa mère; l'impression qui s'en dégage est qu'à ce moment, Sémélé est devenue dans l'idéologie religieuse le faire-valoir de son fils.

En conclusion, il s'agit d'un ouvrage nuancé où l'A. met en évidence les rôles prédominants d'Apollon et d'Artémis (déesse dont la présence est marquée à Sicyone, mais aussi à Corinthe et à Thèbes), auxquels Dionysos se trouve associé en plusieurs endroits, comme si ces trois divinités étaient les acteurs d'un complexe mythico-rituel formant un ensemble solidaire. Cependant, l'A. se préoccupe toujours de souligner les particularités des cultes locaux, les jeux d'influence des traditions ethniques au sein de chacune des cités et l'originalité des stratifications culturelles de ces lieux. Les hypothèses avancées dans cet ouvrage très intuitif sont bien étayées par l'excellente connaissance que l'A. a des sources et de la bibliographie. L'ouvrage se clôt sur un épilogue de Lucia Lamberti consacré aux représentations de Dionysos dans les peintures commandées par Alphonse d'Este à Titien pour la chambre d'albâtre du palais de Ferrare. Bibliographie et index des noms d'auteurs anciens et des noms de divinités et de héros en fin de volume. 